\title{
Corrigendum: Advantage of rare infanticide strategies in an invasion experiment of behavioural polymorphism
}

Tapio Mappes, Jouni Aspi, Esa Koskela, Suzanne C. Mills, Tanja Poikonen \& Juha Tuomi

Nature Communications 3:611 doi: 10.1038/ncomms1613 (2012); Published 3 Jan 2012; Updated 23 Oct 2012

The affiliation details for Jouni Aspi are incorrect in this Article. The correct address for this author is given below.

Department of Biology, University of Oulu, PO Box 3000, FIN-90014 Oulu, Finland. 